\title{
Track cycling: An analysis of the pacing strategies employed during the devil elimination race
}

\section{$\underline{\text { Authors }}$}

1) Kevin. S. Gill - corresponding author.

Affiliations- English Institute of Sport; University of Chester; Harlequins Rugby Union FC. Address: Harlequins Training Centre, Surrey Sports Park, Richard Meyjes Road, Guildford, GU2 7AD. Tel: 07850 716704.Email: kevin.gill@eis2win.co.uk

2) Chris White.

Affiliations- English Institute of Sport. Address: Senior Performance Analyst, English Institute of Sport, Sport City, Gate 13, Rowsley Street, Manchester, M11 3FF. Tel: 0870 759 0466.Email: chris.white@eis2win.co.uk

3) Dr Paul. R. Worsfold.

Affiliations- English Institute of Sport; University of Chester. Address: Head of Sports Biomechanics \& Performance Analysis, University of Chester, Department of Sport \& Exercise Sciences, Parkgate Road, Chester, CH1 4BJ. Tel: +44 (0)1244 513467. Email: p.worsfold@chester.ac.uk

\begin{abstract}
This study aimed to provide a description of the pacing requirements of the track cycling Elimination race, and to identify effective pacing strategies to maximise overall Omnium medal opportunity. Six male, and six female elite competitive races were investigated using half-lap split times. Selected dependant variables were; mean speed and variation in speed. Spearman's Rho correlations were used to test patterns between dependant variables and the final finishing position of riders. One-way ANOVAs were also applied to test for differences in dependant variables, between successful (top 6 finishers) and unsuccessful groups (7th-12th). Pacing patterns of the men's and women's races were complex, but followed an overall positive and variable pacing pattern, with men's race quarter speeds of $52.8 \mathrm{~km} / \mathrm{h}( \pm 1.9), 52.1 \mathrm{~km} / \mathrm{h}( \pm 2.1), 51.1 \mathrm{~km} / \mathrm{h}( \pm 2.2)$, and $49.5 \mathrm{~km} / \mathrm{h}( \pm 5.5)$. In general, differences in pacing strategy were not found to significantly influence the success of riders unless employed late in the race. Results are discussed for the application and the development of effective elimination race strategies and tactics.
\end{abstract}

Key words: Elimination race, track cycling, Omnium, pacing strategies. 


\title{
Track cycling: An analysis of the pacing strategies employed during the devil elimination race
}

\begin{abstract}
This study aimed to provide a description of the pacing requirements of the track cycling Elimination race, and to identify effective pacing strategies to maximise overall Omnium medal opportunity. Six male, and six female elite competitive races were investigated using half-lap split times. Selected dependant variables were; mean speed and variation in speed. Spearman's Rho correlations were used to test patterns between dependant variables and the final finishing position of riders. One-way ANOVAs were also applied to test for differences in dependant variables, between successful (top 6 finishers) and unsuccessful groups (7th-12th). Pacing patterns of the men's and women's races were complex, but followed an overall positive and variable pacing pattern, with men's race quarter speeds of $52.8 \mathrm{~km} / \mathrm{h}( \pm 1.9), 52.1 \mathrm{~km} / \mathrm{h}( \pm 2.1), 51.1 \mathrm{~km} / \mathrm{h}( \pm 2.2)$, and $49.5 \mathrm{~km} / \mathrm{h}( \pm 5.5)$. In general, differences in pacing strategy were not found to significantly influence the success of riders unless employed late in the race. Results are discussed for the application and the development of effective elimination race strategies and tactics.
\end{abstract}

Key words: Elimination race, track cycling, Omnium, pacing strategies. 


\section{Introduction}

The 'Omnium' is a multi-event competition introduced into elite cycling at the 2007 Track Cycling World Championships in Spain. The competition requires riders to compete in a variety of 6 different disciplines. The different disciplines are broadly divided into sprint $(<1000 \mathrm{~m})$ and endurance $(>1000 \mathrm{~m})$ categories (Craig and Norton, 2001). The placing of riders in each discipline is converted into points (e.g. $1^{\text {st }}$ place $=1$ point, $2^{\text {nd }}$ place $=2$ points etc), with the lowest overall point scorer winning the event. The most recent addition to the Omnium is the 'Devil Elimination' race which was first introduced in 2010 at the European Track Championships in Poland. The elimination race involves a maximum of 24 riders, and begins with a rolling start. It is primarily endurance based; however, there is also a sprint element every two laps. At the end of every $2^{\text {nd }}$ lap, the rider who crosses the finish line in last place is eliminated from the race. This continues with a rider eliminated every $2^{\text {nd }}$ lap until one rider remains. The last two riders will complete 48 laps $(12 \mathrm{~km})$. The Elimination race was added to the Omnium in order to give a greater opportunity for endurance riders to achieve a medal (Birnie, 2009). However, research into past medal winners suggests that adding the Elimination race may have had the opposite effect, thereby weighting the race in favour of riders with greater sprint ability (Ofoghi, Zeleznikow, Dwyer, and Macmahon, 2013a).

Due to the nature of the sport, pacing strategies are fundamental to cycling performance and have previously been identified over races varying in distance (Atkinson and Brunskill, 2000; Broker, Kyle, and Burke, 1999; Wilberg and Pratt, 1988; Padilla et al., 2000; Mauger, Jones, and Williams, 2009; Hettinga et al., 2006; De Koning, Bobbert, and Foster, 1999). An athlete's pacing strategy is defined as the distribution of work or 
energy expenditure throughout a time trial, race, or exercise session (Abbiss et al., 2006 Atkinson, Davison, Jeukendrup and Passfield, 2003). However, due to the difficulty of measuring work or energy expenditure, pace is often measured through split times and speeds (Abbiss et al., 2006). A variety of pacing strategies have been identified, including; 'negative pacing', 'positive pacing', 'even pacing', 'parabolic-shaped pacing' and 'variable pacing' (Abbiss and Laursen, 2008; Atkinson, Peacock, and Law, 2007; Liedl, Swain, and Branch, 1999; Swain, 1997). 'Negative pacing' refers to the gradual increase in speed throughout the whole event and is often observed during middle distance events. 'Positive pacing' begins with a fast start, which subsequently, leads to a gradual reduction of pace due to fatiguing effects (Garland, 2005; Abbiss and Laursen, 2008). 'Even pacing', is identified when the athlete maintains a relatively constant energy output throughout the event. Accelerations and decelerations are kept to a minimum as minor fluctuations in speed can lead to greater energy costs (Zamparo et al., 2005; Swain, 1997). Previous analyses of pacing strategies have traditionally used gross measurements over long periods or distances (Van Ingen Schenau, De Koning, and De Groot, 1994). As more precise methods of speed analysis have been applied within sport events, more complex and detailed data of pacing strategies has been acquired (Abbiss et al., 2006; Garland, 2005; Atkinson and Brunskill, 2000). By utilising this data, researchers have shown that during endurance events, athletes often decrease in speed following the start, and then increase speed again towards the end (Abbiss and Laursen, 2008; Garland, 2005). This is known as a 'parabolic-shaped pacing' strategy. In competitions when external conditions fluctuate (for example, wind speed, temperature, humidity, and gradient), 'variable pacing' to match the conditions, has been shown to improve overall performance. 
During cycling races with a large numbers of competitors, it is often difficult for riders to maintain a steady race pace because of the influence of other riders. The rules of the race can also affect the pace, for example, within the elimination race, riders must avoid last position at the end of the elimination lap. Often, the races speed up and slow down, in a constant state of flux, as riders make sprints for the finishing line to avoid elimination. The pack of riders also stretches as these sprints are made, and then compresses back together afterwards acting as a dynamical, self-organising system (Waldron, Worsfold, White and Murray, 2011). Past research into the Omnium has offered an insight into the target finishing positions for riders across the different disciplines, which have historically led to an overall medal win in the event (Ofoghi et al, 2013a; Ofoghi, Zeleznikow, MacMahon, and Dwyer, 2013b; Ofoghi, Zeleznikow, MacMahon, and Dwyer, 2010). However, little is known about how riders can maximise their chances to achieve these positions within the different disciplines, particularly in the complex bunch races. At present little is known about the pacing strategies and tactics of elite cyclists competing within the Elimination race. Therefore the aims of the study were threefold; firstly to identify the overall pacing strategy of elite male and female riders across all the combined elimination races, secondly to identify any differences between the pacing of individual races, and finally to identify if differences in the pacing strategies employed by individual riders within races could impact upon their success within the races.

\section{Methods}

\subsection{Study Design}


Following ethical approval, footage from 12 Elimination International Cycling Union (UCI) races ( 6 male and 6 female) were analysed. The footage included four World Cups, one European Championships and one World Championships. Overall, the pace of the female races were between $6-7 \mathrm{~km} / \mathrm{h}$ slower than the male races, therefore, the male and female data was analysed separately. The top 12 finishers from each race were included in the analysis (male $n=72$, female $n=72$ ).

Pacing during cycling is often analysed using power metres affixed directly to the bike. Data obtained from a power metre would have been preferable in this study, as it provides the direct power output from the rider, and is a more precise measurement of individual pacing. However, this data could not be obtained due to race restrictions; therefore, speed was the only measurement available to analyse pacing during the races. A $25 \mathrm{~Hz}$ video camera was placed on a tripod high in the stands, parallel with the pursuit lines (Figure 1). Two measurements of speed were taken each lap for every rider. The first measurement was calculated from the instant that the centre of the riders rear wheel crossed the finish line, to the opposite pursuit line. The second measurement was taken from the opposite pursuit line, back to the finish line. The time taken by the riders between these two points was collected post-race using computerised analysis software (Dartfish TeamPro, 4.0.9.0 Switzerland), and analysed within Excel (Microsoft, USA). 


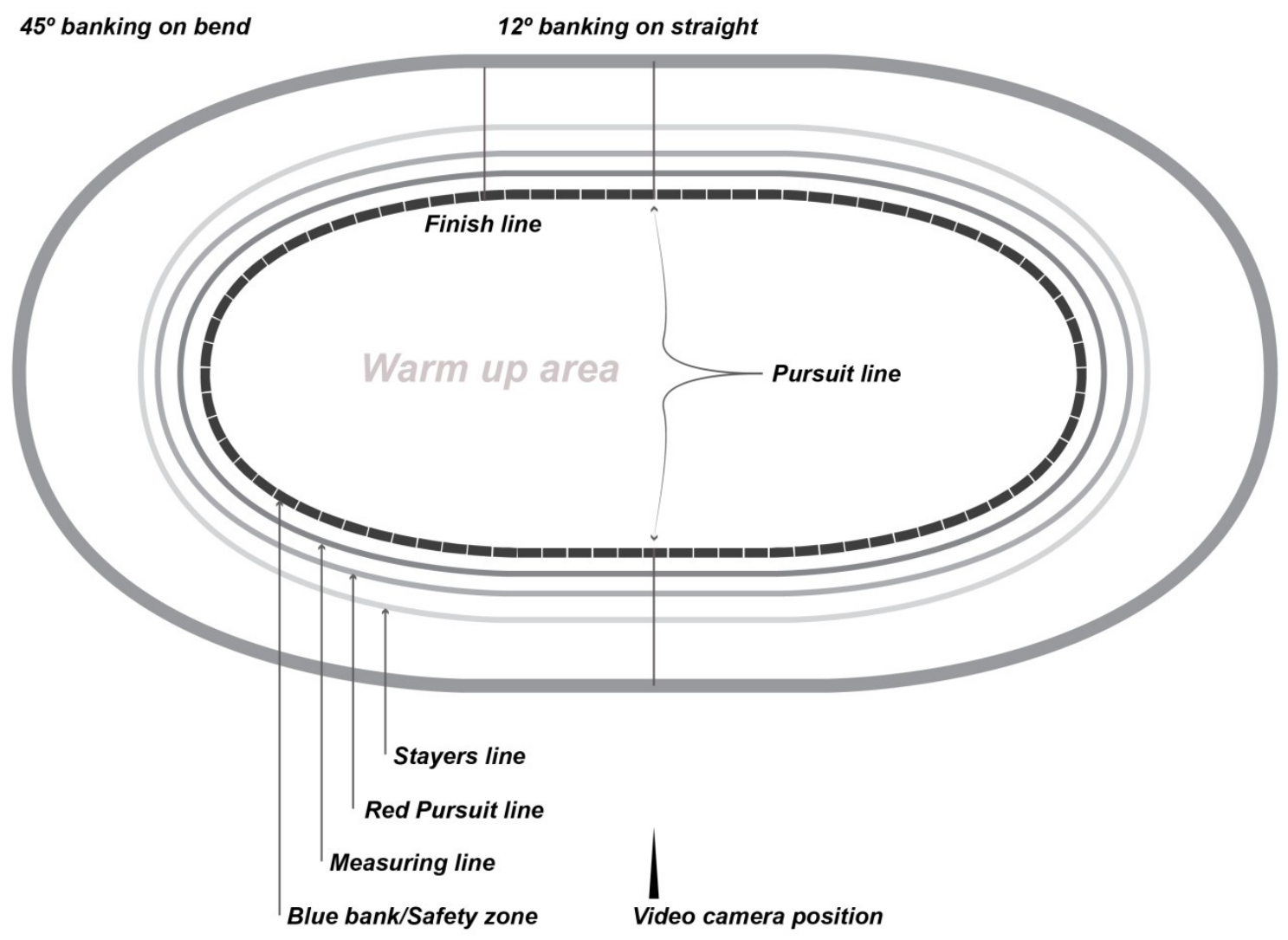

Figure 1. Diagram of velodrome track configuration and camera position.

\subsection{Data analysis}

Preliminary analysis of the speed data, identified patterns, which justified clustering the lap portions into $1^{\text {st }}$ half pre-elimination lap (PE1), $2^{\text {nd }}$ half pre-elimination lap (PE2), $1^{\text {st }}$ half elimination lap (E1), and $2^{\text {nd }}$ half elimination lap (E2), and calculating the mean values for each rider, across each quarter. Initially, a means based analysis of past medal winners within the Omnium was conducted, and it was identified that elite male and female riders must attain at least $6^{\text {th }}$ place in the Elimination race, in order to achieve an overall Omnium medal. This finding was mirrored by that of previous research into the Omnium (Ofoghi et al., 2013a; Ofoghi et al., 2013). Therefore, the riders were divided 
into two equal groups of successful $\left(1^{\text {st }}-6^{\text {th }}\right.$ place $)$ and unsuccessful riders $\left(6^{\text {th }}-12^{\text {th }}\right.$ place $)$. Riders finishing $13^{\text {th }}$ or lower were not included within the analysis. The overall pacing requirements of the races will subsequently be shown as the mean speed of the top 12 finishers. It should be noted, that due to the elimination nature of the races, the number of riders included in the data decreased as the race progressed and riders were eliminated

For analysis of group differences $\left(1^{\text {st }}-6^{\text {th }}\right.$, and $7^{\text {th }}-12^{\text {th }}$ finishing groups $)$ and correlations, the riders' speed and standard deviation (SD) of speed were the selected dependant variables. The independent variable was the official finishing position of each rider. Following tests for normality (Shapiro Wilks $>0.05)$, rider speed $(\mathrm{km} / \mathrm{h})$ was not found to be normally distributed across the majority of lap segments. Therefore, the data was analysed for statistical significance using Spearman's Rho correlation tests. The strength of correlations was based upon; 0.1 or less $=$ no correlation, 0.1 to $0.3=$ weak, 0.3 to $0.5=$ moderate, and 0.5 to $1=$ strong correlation (Choudhury, 2009). In addition, a oneway Analysis of Variance (ANOVA) was used to test for differences in speed and SD of speed, between the two selected groups $\left(1^{\text {st }}-6^{\text {th }}\right.$ place, and $7^{\text {th }}-12^{\text {th }}$ place finishers $)$.

\subsection{Reliability}

Inter and intra-reliability was tested using the limits of agreement method as described by Cooper, Hughes, O'Donoghue, and Nevill (2007). Following pilot testing, it was deduced that $95 \%$ of the test and re-test data should lie within $0.2 \mathrm{~km} / \mathrm{h}$ for the speed variable. Intra-observer reliability tests were conducted on five randomly selected riders, two months apart. Inter-reliability was also analysed for five random rider 
performances, with the re-test performed by a senior performance analyst at the English Institute of Sport. Results for inter and intra-reliability limits of agreement test of the speed variable are shown in table 1.

Table 1. Inter and intra-reliability results comparing the test and re-test for speed.

Rider

Exact match $\leq 0.1 \mathrm{~km} / \mathrm{h}$ Difference $\leq 0.2 \mathrm{~km} / \mathrm{h}$ Difference

\begin{tabular}{lllll|lllll}
\multicolumn{3}{l}{ Inter-reliability \% } & & & \multicolumn{6}{l}{ Intra-reliability \% } \\
\cline { 1 - 6 } $\mathbf{1}$ & $\mathbf{2}$ & $\mathbf{3}$ & $\mathbf{4}$ & $\mathbf{5}$ & $\mathbf{1}$ & $\mathbf{2}$ & $\mathbf{3}$ & $\mathbf{4}$ & $\mathbf{5}$ \\
55.7 & 54.5 & 59.5 & 61.3 & 56.9 & 51.1 & 48.9 & 50 & 45 & 55.3 \\
96.6 & 95.5 & 95.2 & 96.3 & 93.1 & 96.6 & 96.6 & 100 & 98.8 & 97.4 \\
\cline { 5 - 7 } 100 & 100 & 100 & 100 & 100 & 100 & 100 & N/A & 100 & 100
\end{tabular}

The intra-reliability of the speed variable was found to exceed the required $0.2 \mathrm{~km} / \mathrm{h}$ difference, as over $95 \%$ of the differences were within $0.1 \mathrm{~km} / \mathrm{h}$. The inter-reliability of the data for the speed variable was found to meet the requirements of the pre-defined 95\% limits of agreement. Therefore, the testing procedures and methods were deemed to be acceptable. 


\section{Results}

To provide an overview of the pacing of cyclists for the 6 different races, the mean speed of the top 12 finishers for the men's elimination races are shown in table 2.

Table 2. Mean speeds across each quarter for the men's elimination races (Top 12 finishers).

Mean Speed $( \pm$ SD) $(\mathrm{Km} / \mathrm{h})$

\begin{tabular}{llllll}
\hline Race Segment & $\mathbf{1}^{\text {st }}$ Quarter & $\mathbf{2}^{\text {nd }}$ Quarter & $\mathbf{3}^{\text {rd }}$ Quarter & $\mathbf{4}^{\text {th }}$ Quarter & Overall \\
\hline European Champs & $52.5( \pm 1.4)$ & $52.3( \pm 2.4)$ & $51.4( \pm 1.8)$ & $50.6( \pm 4.6)$ & $51.7( \pm 3)$ \\
\hline World Cup 1 & $53.3( \pm 1.9)$ & $52( \pm 1.7)$ & $51.5( \pm 1.8)$ & $49.6( \pm 5)$ & $51.6( \pm 3.2)$ \\
\hline World Cup 2 & $52.7( \pm 2.3)$ & $52.3( \pm 2.2)$ & $51.1( \pm 2.1)$ & $49.2( \pm 4.5)$ & $51.4( \pm 3.2)$ \\
\hline World Cup 3 & $52.1( \pm 2.2)$ & $51.5( \pm 2.4)$ & $50.4( \pm 2.4)$ & $49.2( \pm 5.9)$ & $50.8( \pm 3.7)$ \\
\hline World Cup 4 & $52.7( \pm 1.6)$ & $52.3( \pm 1.8)$ & $51.1( \pm 1.8)$ & $49.8( \pm 5.7)$ & $51.5( \pm 3.3)$ \\
\hline World Champs & $53.4( \pm 1.7)$ & $52.3( \pm 1.9)$ & $51( \pm 3)$ & $48.5( \pm 7.3)$ & $51.55( \pm 5)$ \\
\hline All Races & $52.8( \pm 1.9)$ & $52.1( \pm 2.1)$ & $51.1( \pm 2.2)$ & $49.5( \pm 5.5)$ & $51.4( \pm 3.5)$
\end{tabular}

Figure 2 shows the mean speeds for each quarter of the race taken over each half lap. The white areas of figure 2 contain the pre-elimination laps and the grey shaded area contains the elimination lap. The data for all the men's races combined, showed a linear decrease and a greater variation of speed, as the races progressed (Figure 2). The speeds measured during the second, third, and fourth quarters, tended to follow a similar pattern across all of the races (Table 2). Generally, there was a gradual increase in speed from lowest during PE1, to highest during PE2. This differed slightly during the final quarter of races when speeds during PE2 were usually slower than the PE1 (with the exception of the European Championships) (Table 2). The mean speeds across each 
quarter of all races showed a gradual decrease from the first quarter $(52.8 \mathrm{~km} / \mathrm{h})$ to last quarter $(49.5 \mathrm{~km} / \mathrm{h})$. The fastest starting quarter was during the World Championships, and slowest during World Cup 1. The variation of pace (based on the standard deviation), remained relatively consistent throughout the first three quarters of each race, before increasing notably during the final quarter. The World Championships race had the greatest variation of speed in the final quarter $( \pm 7.3 \mathrm{~km} / \mathrm{h})$, and differed slightly from the other competitions, with a notable increase in variation of speed from the $2^{\text {nd }}$ $( \pm 1.9 \mathrm{~km} / \mathrm{h})$ to the $3^{\text {rd }}$ quarter $( \pm 3 \mathrm{~km} / \mathrm{h})$ (Table 2$)$.

Figure 2. Race quarter mean speeds (with standard deviation and linear trendline) collected for each half lap of the Elimination race for all six men's competitions combined (Top 12 finishers).

The Spearman's Rho correlation co-efficient values for mean speed and speed SD (versus finishing position), across the combined men's races, are shown in figure 3 and 4 respectively. Across the first two quarters of races, very low correlation scores indicated no relationship between the speed variable and the final finishing position (Figure 3). Negative correlations for the speed variable (indicating lower relative speed was related to better finishing position) were found to be weak across the $3^{\text {rd }}$ quarter PE2 $(-0.27, \mathrm{P}<0.05)$, moderate across the $4^{\text {th }}$ quarter PE1 $(-0.47, \mathrm{P}<0.01)$, and strong 
across the $4^{\text {th }}$ quarter PE2 $(-0.54, \mathrm{P}<0.01)$. Weak (not significant) positive correlations (indicating higher relative speed was related to better finishing position) were found across the $3^{\text {rd }}$ quarter E2 $(0.17)$, and the $4^{\text {th }}$ quarter E1 and E2 $(0.22,0.27$; respectively).

Correlations for the speed SD showed weak or no correlations up until the end of the $3^{\text {rd }}$ quarter (Figure 4). Over the $4^{\text {th }}$ quarter, moderate positive correlations were found for the speed SD variable (indication that higher relative variation of speed was related to better overall finishing position) across PE1 and PE2 $(0.45, \mathrm{P}<0.05 ; 0.44, \mathrm{P}<0.05$, respectively), and E1 and E2 (0.31; 0.36, respectively, not significant). Results of the one-way ANOVAs performed on the men's speed and speed SD variables showed no significant difference between successful and unsuccessful groups, and therefore, this data has not been displayed.

Figure 3. Spearman's Rho value for mean speed against final race finishing position in the men's Elimination races. Positive value $=$ higher speed correlated with better/lower finishing position. Significant correlations denoted as $*=\rho<0.05 ; * *=\rho<0.01$.

Figure 4. Spearman's Rho (and significance value) for speed SD against final race finishing position in the men's Elimination races. Positive value $=$ higher speed variation 
correlated with better/lower finishing position. Significant correlations denoted as $*=\rho$ $<0.05 ; * *=\rho<0.01$.

The mean speed of the top 12 finishers for the combined women's elimination races is shown in figure 5 , and table 3 . The data showed a gradual decrease in speed from $1^{\text {st }}$ quarter $(48.4 \mathrm{~km} / \mathrm{h} \pm 1.7)$ to last quarter $(46 \mathrm{~km} / \mathrm{h} \pm 3.1)$, in a similar pattern to the men's competition. The speeds across the elimination laps increased from E1 to E2, as was observed for the men's competition. However, the change in speed from PE1 to PE2 varied between race quarters, with an increase across the $1^{\text {st }}$ and $2^{\text {nd }}$ quarters, and a decrease across the $3^{\text {rd }}$ and $4^{\text {th }}$ quarters.

Figure 5. Race quarter mean speeds (with standard deviation and linear trendline) collected for each half lap of the Elimination race for all six women's competitions combined (Top 12 finishers).

Table 3. Mean speeds across each quarter for the women's Elimination races (Top 12 finishers).

Mean Speed $( \pm$ SD) $(\mathbf{K m} / \mathbf{h})$

\begin{tabular}{llllll}
\hline Race Segment & $\mathbf{1}^{\text {st }}$ Quarter & $\mathbf{2}^{\text {nd }}$ Quarter & $\mathbf{3}^{\text {rd }}$ Quarter & $\mathbf{4}^{\text {th }}$ Quarter & Overall \\
\hline European Champs & $47.8( \pm 1.3)$ & $47.2( \pm 1.5)$ & $48.1( \pm 1.6)$ & $45.8( \pm 2.4)$ & $47.2( \pm 1.9)$ \\
\hline World Cup 1 & $48.4( \pm 1.6)$ & $47.2( \pm 2)$ & $47.4( \pm 1.9)$ & $46.6( \pm 2.7)$ & $47.4( \pm 2.2)$ \\
\hline World Cup 2 & $49.6( \pm 1.8)$ & $48.1( \pm 1.9)$ & $47.1( \pm 1.7)$ & $45.1( \pm 4.4)$ & $47.5( \pm 3.1)$ \\
\hline World Cup 3 & $49( \pm 2.1)$ & $48.1( \pm 1.2)$ & $47.5( \pm 1.9)$ & $47.4( \pm 2.3)$ & $48( \pm 2)$ \\
\hline
\end{tabular}




\begin{tabular}{llllll}
\hline World Cup 4 & $47.7( \pm 1.7)$ & $48( \pm 2.1)$ & $47.3( \pm 2)$ & $46.3( \pm 2.1)$ & $47.3( \pm 2.1)$ \\
\hline World Champs & $48( \pm 1.5)$ & $47.2( \pm 1.3)$ & $45.9( \pm 1.7)$ & $45.3( \pm 4.7)$ & $46.6( \pm 2.8)$ \\
\hline All Races & $48.4( \pm 1.7)$ & $47.6( \pm 1.7)$ & $47.2( \pm 1.8)$ & $46( \pm 3.1)$ & $47.3( \pm 2.4)$
\end{tabular}

Across all women's races combined, the variation of speed (based upon the standard deviation) tended to remain stable across the first three quarters, then showed a marked increase during the final quarter. As was found for the men's competition, the highest variation of pace was found across the final quarter of the women's World Championship Elimination race $( \pm 4.7 \mathrm{~km} / \mathrm{h})$.

The Spearman's Rho correlation co-efficient values, for mean speed and speed SD (versus finishing position) across the combined women's races, are shown in figure 6 and 7 respectively. For all the races combined, no correlations were found for the speed and speed SD variables, across the first 3 quarters, indicating no relationship with finishing position. For the speed variable, a negative correlation (indicating lower relative speed was related to better finishing position) was found over the $4^{\text {th }}$ quarter PE2 (-0.33, not significant), and a positive correlation (indicating higher relative speed was related to better finishing position) was found over the $4^{\text {th }}$ quarter of E2 $(0.36$, not significant). For the speed SD variable, strong positive correlations (indicating higher relative variation of speed was related to better finishing position) were found over the $4^{\text {th }}$ quarter of PE2 $(0.51, \mathrm{P}<0.01)$, and the $4^{\text {th }}$ quarter of E1 $(0.64, \mathrm{P}<0.01)$, and a moderate positive correlation was found over the $4^{\text {th }}$ quarter of E2 $(0.372$, not significant). Significant positive correlations over the $4^{\text {th }}$ quarter tended to indicate similar patterns to the men's races, with faster speeds over the pre-elimination lap linked to a worse finishing position. As was found for the men's races, results of the one-way ANOVAs performed on the speed and speed SD variables showed no significant 
difference between successful and unsuccessful groups, and therefore the data has not been displayed.

Figure 6. Spearman's Rho (and significance value) for mean speed against final race finishing position in the women's Elimination races. Positive values $=$ higher speed correlated with better/lower finishing position. Significant correlations denoted as $*=\mathrm{P}$ $<0.05 ; * *=\mathrm{P}<0.01$.

Figure . Spearman's Rho (and significance value) for speed SD against final race finishing position in the women's Elimination races. Positive values $=$ higher variation of speed correlated with better/lower finishing position. Significant correlations denoted as $*=\rho<0.05 ; * *=\rho<0.01$.

\section{Discussion}

The aim of this study was to identify the pacing strategies used in the elimination race, and how differences in pacing can influence the success of the riders. Overall, for men's and women's races, it was found that the mean speed of the races gradually reduced as they progressed, with a quick start, as opposed to a constant steady pace. This suggests that the riders followed an overall 'positive pacing' strategy (Abbiss and Laursen, 2008). However, over the first three quarters of the race, the variation of pace was between $3 \%$ and $5 \%$ of the mean pace. This type of disparity in pace $(5 \%)$ has been 
defined as a 'variable pacing' strategy in previous research (Atkinson et al., 2007; Liedl et al., 1999, Swain, 1997). Furthermore, whilst the mean speed was slower during the $4^{\text {th }}$ quarter, the variation of speed was greater (6-8\% variance for women, and $10-15 \%$ for men), which indicated more intense sprints. This suggests that the effort of riders remains high, due of the energy cost of an increased variance of speed (Zamparo et al., 2005; Swain, 1997). Subsequently, the work performed by athletes could have been greater over the final quarter, because of the accelerations required during the sprints (Abbiss et al., 2006; Atkinson et al., 2003; De Koning, Bobbert and Foster, 1999). Therefore, in terms of work or energy, it is likely that an overall 'parabolic-shaped pacing' strategy is used within the elimination race (Abbiss and Laursen, 2008; Garland, 2005). However, further analysis measuring physiological responses during the race would be required in order to examine the pacing strategy in more detail. Interestingly, the pace data for the World Championships, indicated a faster starting pace, with more intense sprints in the final quarter. This suggests that the World Championships is more demanding, in terms of work and energy requirements, than the European Championships and World Cups. The higher demand during these races is likely the result of the weighting of qualification points, which makes the World Championships more important to future qualification and Olympic selection, and also the general perception that it is a more prestigious event. This usually results in a higher number of world class athletes taking part, but also leads athletes to prepare more thoroughly for the event by structuring their training programmes, so that they can achieve peak performance levels.

The one-way ANOVA tests revealed no significant differences in pace, between successful and unsuccessful groups for the men's or women's races. Furthermore, 
correlation scores indicated that differences in mean speed did not benefit riders significantly (in terms of final finishing position) until the $3^{\text {rd }}$ and $4^{\text {th }}$ quarters. Therefore, variation in pacing strategies between riders does not appear to affect the success of riders until later in the races. Over the $3^{\text {rd }}$ and $4^{\text {th }}$ quarter of races, male and female riders may gain some benefit from slower speeds during the pre-elimination lap (particularly towards the end of the lap), and faster speeds during the elimination lap (relative to the top 12 mean speed). Correlation scores for SD of speed and final finishing position suggested that for both men's and women's races, a high variation of pace over the $4^{\text {th }}$ quarter (relative to the pack of riders), was linked to success. Therefore, training should be based around a highly variable pacing strategy late in the race (Atkinson et al., 2007; Liedl et al., 1999, Swain, 1997). Track cycling teams should set gearing with ratios that allow athletes to maintain the overall pace of the race, but also account for the variations in pace, which become increasingly more severe as the race progresses.

\subsection{Future Directions}

The methods used to examine the effectiveness of different strategies employed within races, does not take into account the differing ability of the riders. Riders with a superior physical or psychological ability may be able to employ any tactic, or no precise tactic, and still perform well in the race. Future studies should look to normalise data in accordance to previous results in order to identify effective strategies for riders with higher or lower ability. Furthermore, this study examined strategies by means of examination of in-race speed data alone, and therefore, it cannot be ascertained if the strategies were employed consciously by any of the riders. Future studies looking to examine strategies would benefit from confidential interviews or questionnaires with 
riders prior to races, to examine which strategies they will be attempting to employ. This would allow for a more precise examination of the success of different strategies, and how various strategies interact between riders. Further research into the spatial aspect of the race would also be warranted, as this could enlighten riders and coaches to the most effective in-race positional strategies, which could be implemented to maximise the chance of a medal win.

\section{Conclusion}

The pacing strategies observed during races was found to be highly complex, and contained aspects of 'positive', 'parabolic-shaped', and 'variable' pacing strategies. The results suggest that differences in racing strategies can be quantitatively measured, and that the subsequent impact upon success can be assessed. It is envisaged that future research can build upon the findings of the current study, and further develop our understanding of the pacing strategies and tactics employed during the Elimination race (and cycling bunch races in general), from an objective and quantitative perspective.

\section{Acknowledgement}

The authors wish to thank Sam Barnes, for provision of the velodrome track diagram.

\section{References}


Abbiss, C. R., and Laursen, P. B. (2008). Describing and understanding pacing strategies during athletic competition. Sports Medicine, 38(3), 239-252.

Abbiss, C. R., Quod, M. J., Martin, D. T., Netto, K. J., Nosaka, K., Lee, H., Suriano, R., Bishop, D., and Laursen, P. B. (2006). Dynamic pacing strategies during the cycle phase of an ironman triathlon. Medicine \& Science in Sports \& Exercise, $38(4), 726-734$.

Atkinson, G., and Brunskill, A. (2000). Pacing strategies during a cycling time trial with simulated headwinds and tailwinds. Ergonomics, 43(10), 1449-1460.

Atkinson, G., Davison, R., Jeukendrup, A., and Passfield, L. (2003). Science and cycling: Current knowledge and future directions for research. Journal of Sports Sciences, 21, 767-787.

Atkinson, G., Peacock, O., and Law, M. (2007). Acceptability of power variation during simulated hilly time trial. International Journal of Sports Medicine, 28(2), $157-163$.

Birnie, L. (2009, December 17). Cycling Weekly. Retrieved from: http://www.cyclingweekly.co.uk/news/latest/435675/olympic-Omnium-formatunveiled.html

Broker, J. P., Kyle, C. R., and Burke, E. R. (1999). Race cyclist power requirements in the 4000-m individual and team pursuits. Medicine \& Science in Sports \& Exercise, 31(11), 1677-1685.

Choudhury, A. (2009). Statistical correlation. Retrieved from experimental resources web site: $\underline{\text { http://www.experiment-resources.com/statistical-correlation.html }}$

Cooper, S., Hughes, M., O'Donoghue, P., and Nevill, A. M. (2007). A simple statistical method for assessing the reliability of data entered into sport performance 
analysis systems. International Journal of Performance Analysis in Sport, 7(1), 87-109.

Craig, N. P., and Norton, K. I. (2001). Characteristics of track cycling. Sports Medicine, 31(7), 457-468.

De Koning, J. J., Bobbert, M. F., and Foster, C. (1999). Determination of optimal pacing strategy in track cycling with an energy flow model. Journal of Science and Medicine in Sport, 2(3), 266-277.

Garland, S. W. (2005). An analysis of the pacing strategy adopted by elite competitors in 2000m rowing. British Journal of Sports Medicine, 39, 39-42.

Hettinga, F. J., De Koning, J. J., Broersen, F. T., Van Geffen, P., and Foster, C. (2006). Pacing strategy and the occurrence of fatigue in 4000-m cycling time trials. Medicine \& Science in Sports \& Exercise, 38(8), 1484-1491.

Liedl, M. A., Swain, D. P., and Branch, J. D., (1999). Physiological effects of constant versus variable power output during endurance cycling. Medicine $\&$ Science in Sports \& Exercise, 31(10), 1472-1477.

Mauger, A. R., Jones, A. M., and Williams, C. A. (2009). The effect of non-contingent and accurate performance feedback on pacing and time trial performance in 4km track cycling. British Journal of Sports Medicine, 45, 225-229.

Ofoghi, B., Zeleznikow, J., Dwyer, D., and MacMahon, C. (2013a). Modelling and analysing track cycling Omnium performances using statistical and machine learning techniques. Journal of Sport Sciences, 31(9), 954-962. 
Ofoghi, B., Zeleznikow, J., MacMahon, C., and Dwyer, D. (2010). A machine learning approach to predicting winning patterns in track cycling omnium. Artificial Intelligence in Theory and Practice, 331, 67-76.

Ofoghi, B., Zeleznikow, J., MacMahon, C., and Dwyer, D. (2013b). Supporting athlete selection and strategic planning in track cycling omnium: A statistical and machine learning approach. Information Sciences, 233, 200-213.

Padilla, S., Mujika, I., Angulo, F., and Goiriena, J. J. (2000). Scientific approach to the 1-h cycling world record: A case study. Journal of Applied Physiology, 89(6), $1522-1577$.

Swain, D. P. (1997). A model for optimizing cycling performance by varying power on hills and in wind. Medicine \& Science in Sports \& Exercise, 29(8), 1104-1108.

Van Ingen Schenau, G. J., De Koning, J. J., and De Groot, G. (1994). Optimisation of sprinting performance in running, cycling, and speed skating. Sports Medicine, 17(4), 259-275.

Waldron, M., Worsfold, P., White, C., and Murray, S. (2011). Swarming behaviour in elite race bunch cycling: A case study. International Journal of Performance Analysis in Sport, 11(1), 14-25.

Wilberg, R. B., and Pratt, J. (1988). A survey of the race profiles of cyclists in the pursuit and kilo track events. Canadian Journal of Sports Science, 13(4), 208213.

Zamparo, P., Bonifazi, M., Faina, M., Milan, A., Sardella, F., Schena, F., and Capelli, C. (2005). Energy cost of swimming of elite long-distance swimmers. European Journal of Applied Physiology, 94(5-6), 697-704. 